\title{
Predicting recurrence of depression
}

\author{
Paul Kurdyak MD PhD, John Cairney PhD
}

See related research article by Colman and colleagues on page 1969 and at www.cmaj.ca/lookup/doi/10.1503/cmaj.110676

$\mathrm{D}$ epression is widely viewed as a chronic and recurrent mental illness that carries a high burden of illness, which is mostly related to a young age at onset and chronicity of illness. ${ }^{1}$ However, not everyone who recovers from a first episode of depression will have a subsequent episode. In a related research article, Colman and colleagues used population-based longitudinal data to try to identify risk factors that predict recurrence. ${ }^{2}$

Individuals with recurrent depression tend to have more severe initial episodes and a poorer response to treatment during subsequent recurrences compared with those without recurrent depression. ${ }^{3}$ One goal of predicting which patients are likely to experience a recurrence is to identify those who should receive long-term treatment for depression. Although this is logical, predicting recurrence is a formidable challenge. The inability to predict recurrence with confidence means that we lack the tools to apportion resources to those who have the greatest need. More research is required to better understand the natural history of the disorder - including its recurrence - to guide clinical practice.

Colman and colleagues used longitudinal survey data from the National Population Health Survey, which has been administered to a cohort every two years since 1994/95. This survey includes questions from a depression rating scale, which enabled the authors to model recurrence. Of the respondents, 585 reported experiencing depression during the baseline year (2000/01) and had variable courses of recurrence during the follow-up period. Despite including many variables in the analysis, the authors found that only three predicted recurrence in the multivariable regression: history of depression, daily smoking and a low level of mastery (a psychological concept that describes a sense of control over one's life and experiences). History of depression was, by far, the strongest predictor of recurrence.

There may be two reactions to this study. One reaction is to believe that the data in this longitudinal survey are not comprehensive enough to allow us to identify factors that accurately predict depression. The other reaction is to believe that there is no method beyond that used by Colman and colleagues that can add to our ability to predict recurrence. $^{2}$

Data from population-based surveys may be useful for understanding the predictors of recurrence because the samples tend to be more heterogeneous than those derived from clinical populations and therefore may potentially be more representative of the population with the disorder. At the same time, such heterogeneity can lead to at least two major limitations. First, it may be harder to study recurrence because there will be fewer people with severe depression (and many more with mild to moderate depression) in nonclinical samples. Less severity of depression in the sample population will reduce the incidence of recurrence in the sample. Second, longitudinal surveys assess individuals at regular intervals (e.g., every two years in the National Population Health Survey). These intervals are not necessarily selected to correspond with the recurrence of depressive episodes, thus there is a good chance that even with frequent assessment, respondents will need to rely on their recall of depressive episodes.

The National Population Health Survey included few measures that might help us to better understand recurrence. Depression, like all mental illnesses, is believed to have a biopsychosocial cause. There is little in the way of biological or psychological measures in this survey. Those who do not believe that this survey is sufficient would likely want a follow-up study that follows a population-based cohort of individuals from incident episode of depression.

\section{- Ker points}

- Predicting which patients have a recurrent form of depression is challenging.

- Studying all variables that could predict recurrence of depression is difficult because it is a biopsychosocial illness.

- Increased severity of illness and a history of depression are the strongest predictors of recurrence.

- Strategies to manage recurrent depression should target patients with severe and recurrent forms of depression in the absence of more refined predictors. 
Such a follow-up study should also include biological markers (genetic tests) and more comprehensive psychological measures.

What would be an appropriate reaction to those who believe that the study accurately and comprehensively describes the recurrence of depression? The most robust predictors of recurrence were severity of illness at first presentation and a history of depression. Smoking and low mastery are also predictors, but it is unclear how to use this information to change the trajectory of depression. Perceived mastery may be tapping into a personality or resiliency trait that could be targeted clinically. Unfortunately, mastery is a global measure of control, so it is difficult to ascertain what is actually being captured with this measure. The utility of smoking as a predictor of depression is less clear.

The response to clinical severity and recurrence from a health system perspective is to apportion resources to individuals who are severely ill, especially at first presentation, and to those who have had a recurrence. This would appear to be an appropriate strategy to manage depression (indeed, it is an appropriate strategy for any disease or condition). Unfortunately, the reality for most people with depression is that access to specialty care is problematic and treatment options beyond psychopharmacology are scarce.

Until we have the equivalent of the Framingham rules for assessing cardiovascular risk to predict the risk of depression, we should stick to what we know: people with severe and recurrent depression need access to comprehensive strategies to help manage the very real risk of poor quality of life and depression-related burden of illness.

\section{References}

1. World Health Organization. Depression. Geneva [Switzerland]: The Organization; 2011. Available: www.who.int/mental_health /management/depression/definition/en/ (accessed 2011 Sept. 25).

2. Colman I, Naicker K, Zeng Y, et al. Predictors of long-term prognosis of depression. CMAJ 2011;183:1969-76.

3. Fekadu A, Wooderson S, Markopoulo K, et al. What happens to patients with treatment-resistant depression? A systematic review of medium to long-term outcome studies. J Affect Disord 2009;116:4-11.

Affiliations: Paul Kurdyak and John Cairney are with the Centre for Addiction and Mental Health, and the Institute for Clinical Evaluative Sciences, Toronto, Ont.; John Cairney is with the Departments of Family Medicine, and Psychiatry and Behavioural Neurosciences, McMaster University, Hamilton, Ont.

Contributors: Both authors contributed to drafting and revising the manuscript and approved the final version submitted for publication. 